\title{
Optical Studies of Non-Magnetic Spacer in Thin Fe/Si Multilayers
}

\author{
W. Szuszkiewicz ${ }^{a, *}$, M. Jounnne ${ }^{b}$, J.F. Morhange ${ }^{b}$, \\ M. Chernyshova ${ }^{a}$, L. KowalczyK $^{a}$, E. Łusakowska ${ }^{a}$, \\ P. WANDZIUK ${ }^{c}$ AND T. LUCIŃSKI ${ }^{c}$ \\ ${ }^{a}$ Institute of Physics, Polish Academy of Sciences \\ al. Lotników 32/46, 02-668 Warsaw, Poland \\ ${ }^{b}$ Institut des Nanosciences de Paris, Université Pierre et Marie Curie \\ Campus Boucicaut, 140, rue de Lourmel, 75015 Paris, France \\ ${ }^{c}$ Institute of Molecular Physics, Polish Academy of Sciences \\ Smoluchowskiego 17, 60-179 Poznań, Poland
}

Structures containing magnetic metallic layers attract a lot of attention because of their possible applications in the area of spintronics. The hybrid structures compatible with the Si crystal lattice parameter are of special interest. In this work the short-period $\mathrm{Fe} / \mathrm{Si}$ multilayers were grown by the sputtering onto (001)-oriented Si substrate and investigated by various techniques. After the deposition, all multilayers were characterized by atomic force microscopy. The goal of the present paper was to determine the chemical composition of thin layer created at the interface in $\mathrm{Fe} / \mathrm{Si}$ multilayers due to the Fe diffusion into $\mathrm{Si}$, as well as to analyze the phenomena, which take place in this area. The results of the optical characterization by the Raman scattering were correlated with the magnetic properties of investigated structures (determined by means of the Kerr rotation).

PACS numbers: 68.55.--a, 75.70.-i, 78.30.-j

\section{Introduction}

For many years the structures containing magnetic metallic layers attract a lot of attention because of their possible applications in the area of spintronics: they are considered as principal components of the spin injectors, detectors, or fil-

*corresponding author; e-mail: szusz@ifpan.edu.pl 
ters. In particular, all hybrid structures compatible with the Si lattice parameter are of special interest because they can be easily applied in semiconductor industry. One of the best known and intensively investigated systems of this group is $\mathrm{Fe} / \mathrm{Si}$ and several papers devoted to the properties of $\mathrm{Fe} / \mathrm{Si} / \mathrm{Fe}$ trilayers or $\mathrm{Fe} / \mathrm{Si}$ multilayers were published during the last ten years. Due to the diffusion at the interface the nominally non-magnetic, amorphous $\mathrm{Si}$ spacer in the structures above mentioned is in reality an Fe-Si phase. The problem is that the Fe-Si system has a very rich phase diagram. The magnetic and electric properties of this system strongly depend on its chemical composition. Several more or less direct experiments were performed in order to get precise information about the character of the spacer in $\mathrm{Fe} / \mathrm{Si}$ quantum structures. As it is now clear, the spacer composition is not uniform. Moreover, it strongly depends on several parameters selected during the layer growth or deposition (substrate temperature, deposition rate, etc.). In particular, the interlayer exchange coupling in $\mathrm{Fe} / \mathrm{Si}$ system which is the basic phenomenon deciding about the future applications of such structures, depends in a drastic manner on the chemical composition of the spacer.

The goal of the present work was to get an independent information about possible evolution of the spacer in Fe/Si multilayers with an increasing Fe thickness and to correlate this information with the modification of the magnetic properties of investigated structures. The Raman scattering and Kerr rotation measurements, respectively, were selected for this purpose.

\section{Experimental}

The samples were grown at the Institute of Molecular Physics of the Polish Academy of Sciences in Poznan. The set of a short-period Fe/Si multilayers containing very thin Fe layers was deposited in UHV chamber by DC magnetron sputtering onto oxidized, (001)-oriented Si substrates. The thickness of oxidized Si layer was about $1000 \AA$. The first Fe layer was deposited directly on the top of this oxidized layer. Each sample was composed of 15 periods, the nominal thickness of Fe layers varied between $2.5 \AA$ and $15 \AA$ and that of $\mathrm{Si}$ was fixed at $11 \AA$ (an optimal thickness value corresponding to the highest antiferromagnetic exchange coupling between Fe layers - see, e.g., [1] and references). Every $\mathrm{Fe} / \mathrm{Si}$ multilayer was protected by an additional Fe layer grown on the top of the structure, the thickness of this cap layer was exactly the same as that inside the sample. After the deposition, the $\mathrm{Fe} / \mathrm{Si}$ multilayers were characterized by atomic force microscopy (AFM).

In order to analyze an evolution of the chemical composition of the spacer with an increasing Fe layer thickness, the Raman scattering studies were carried out. These measurements were performed by using Jobin-Yvon U1000 spectrometer equipped with holographic gratings. The Raman spectra were collected at room temperature (RT), using the $514.5 \mathrm{~nm} \mathrm{Ar}^{+}$laser line as excitation. The Raman spectra were recorded in a range of wave numbers from zero to $600 \mathrm{~cm}^{-1}$, 
the spectral resolution was better than $1 \mathrm{~cm}^{-1}$. The Raman spectrum for a $1000 \AA$ thick, amorphous $\mathrm{FeSi}_{2}$ layer deposited by the sputtering at similar technology conditions was also taken for comparison.

The magnetic behaviour of the Fe/Si layers was investigated at RT by the measurement of the Kerr rotation in the longitudinal geometry using an $\mathrm{He}-\mathrm{Ne}$ laser and a photoelastic modulator at a typical modulation frequency of $50 \mathrm{kHz}$. The magnetic field direction was contained in the plane of the layer and its intensity changed from zero to about $2 \mathrm{kOe}$.

\section{Results}

In order to estimate the sample roughness, AFM measurements were carried out. The root mean square (RMS) value of the standard deviation from the average position of the structures at the sample surface was determined on an area equal to one square micrometer for each sample. The results of AFM characterization demonstrated that the surface of the investigated multilayers was rather smooth. However, the RMS value changes in a non-monotonous manner when the Fe layer thickness increases. For multilayers with the shortest periods, the RMS is as low as $3 \AA$, so the surface of these samples is atomically flat (the thickness of one Fe monolayer (ML) is equal to $2.86 \AA$ ). Figure 1 shows the results of AFM measurements performed on Fe/Si multilayer with $3 \AA$ thick Fe layers. The RMS value reaches its maximum (12 $\AA$ ) for the Fe/Si multilayer made of $7 \AA$ thick Fe layers separated by Si layers and later on decreases to the value of $7 \AA$, for $\mathrm{Fe} / \mathrm{Si}$ multilayer with the $15 \AA$ thick Fe.

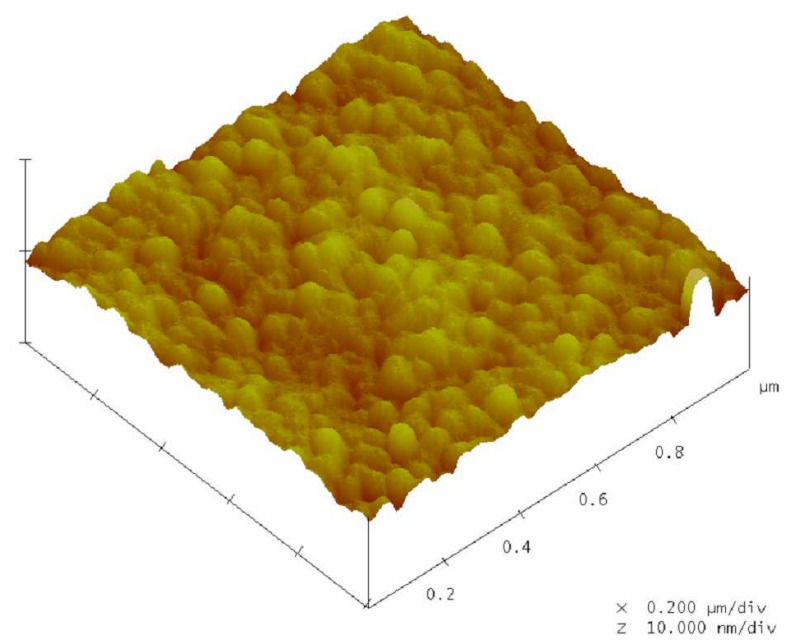

Fig. 1. The result of AFM measurement performed at RT on $\mathrm{Fe} / \mathrm{Si}$ multilayer with $3 \AA$ thick Fe layers. The analyzed area corresponds to one square micrometer, the RMS roughness value is $5 \AA$. 
The analysis of the Raman spectra collected on all samples enables us to get important new information about the investigated system. First of all, the structure due to the excitation of the optical phonon of crystalline Si substrate is observed at $521 \mathrm{~cm}^{-1}$ for all the investigated $\mathrm{Fe} / \mathrm{Si}$ multilayers. This ensures that the values of the Fe layer thickness in multilayers is so thin that the visible light can penetrate inside the whole structures. In other words, if the Raman scattering efficiency of a given phase constituting the structure under consideration is large enough, the presence of this material should be detected as a relevant structure in the Raman spectrum. To our surprise, in spite of a high total thickness of all spacers nominally containing amorphous $\mathrm{Si}$, no trace of this $\mathrm{Si}$ phase was observed in the Raman spectra. Such broad and weak structure, centred at about $480 \mathrm{~cm}^{-1}$ was previously seen by the Raman scattering on similar Fe/Si multilayers [2]. However, the multilayers investigated in [2] were deposited by sputtering under different conditions and in a different UHV chamber and contained more Si because of the higher repetition number in one multilayer. On the contrary, a very broad and weak structure is observed in the Raman spectra collected on the Fe/Si multilayer containing $3.0 \AA$ thick Fe layers as presented in Fig. 2. A sharp structure due to the Raman scattering on Si optical phonon (resulting from the excitation of the substrate) is also seen in this figure. In this figure the phonon density of states of an amorphous $\mathrm{FeSi}_{2}[3]$ is also presented. As can be observed, the Raman spectra match very well with the density of states of $\mathrm{FeSi}_{2}$. This attribution is confirmed by the observation of a similar Raman spectrum taken on an intentionally prepared thick amorphous $\mathrm{FeSi}_{2}$ layer. It must be mentioned that the intensity of this structure does not increase, but in fact decreases when increasing the Fe thickness, and is no more observable as soon as the Fe thickness is larger than $7 \AA$. This behaviour showing a larger signal due to $\mathrm{FeSi}_{2}$ (and thus a larger volume of this material) on the thinnest Fe layer is somehow surprising.
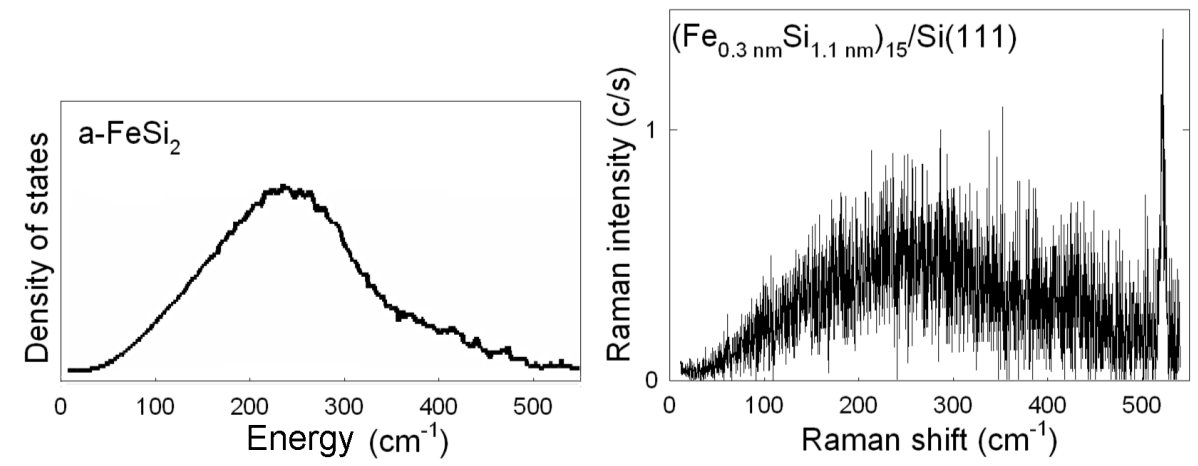

Fig. 2. The comparison of the phonon density of states for an amorphous $\mathrm{FeSi}_{2}$ layer (after [3]) with the Raman spectra collected at RT for Fe/Si multilayers with the Fe layers $3 \AA$ thick. 


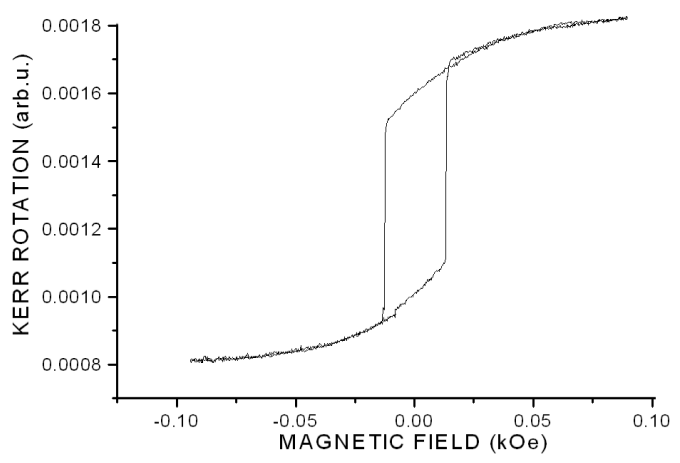

Fig. 3. The magnetization loop measured by the Kerr effect at RT. The Kerr rotation spectrum was collected in the longitudinal geometry on Fe/Si multilayer with $10 \AA$ thick Fe layers.

Concerning the Kerr effect measurements, no magnetic signal could be detected on the $\mathrm{Fe} / \mathrm{Si}$ multilayers for which the $\mathrm{FeSi}_{2}$ Raman signal was observed (and vice versa). For samples containing Fe layers thicker than $7.0 \AA$, magnetization loops are observed and the coercive field strongly increases with the Fe layer thickness. An example of such magnetization loop is shown in Fig. 3.

\section{Discussion}

The results of AFM measurements suggest the change of the Fe growth mode when increasing the thickness of the layer. Below some critical thickness, probably corresponding to about $3 \mathrm{ML}$, deposited Fe does not create an uniform Fe layer but is randomly distributed on the surface in the form of small, separated islands. Such growth mode of the layer is known as the Volmer-Weber type. The sample surface roughness is then at the highest value just below the critical thickness of the deposited layer. Above the critical thickness a more uniform epitaxial Fe layer appears which results in the decrease in the RMS roughness value.

This behaviour is in qualitative agreement with the recent report of the growth of single, thin Fe films deposited on (111)-oriented Si substrate covered by thin iron silicide layer [4]. According to that paper, for a nominal thickness of $1 \mathrm{ML}$ the Fe islands cover less than $20 \%$ of the sample surface, these islands start to coalesce around 3.5 ML, and for a coverage larger than $5 \mathrm{ML}$, all the Fe islands percolate to cover the entire surface. The RMS roughness value, reported in [4], increased from about $5 \AA$ to about $10 \AA$ for the Fe thickness range corresponding to the present studies. Moreover, onset of the long-range ferromagnetic order determined in the same paper by the magnetization Kerr loops collected in polar and longitudinal geometries, occurred at 4.7 ML Fe coverage.

It is not excluded that in our case the relevant Fe thickness values are slightly smaller because of different conditions during the deposition process. Moreover, due to an additional interlayer exchange coupling mechanism (which does not exist 
for single Fe layers reported in [4]), analyzed by us, Fe/Si multilayers could exhibit the ferromagnetic properties for thinner Fe layers.

For thinner samples, Fe deposited in clusters easily reacts with its silicon environment which results in formation of $\mathrm{FeSi}_{2}$. For thicker samples, Fe layers are continuous and the formation of $\mathrm{FeSi}_{2}$ is only achieved at the interface of $\mathrm{Fe}$ and $\mathrm{Si}$. In summary, the quantity of $\mathrm{FeSi}_{2}$ is probably smaller in the last case because the surface of interaction is reduced and the Raman intensity of $\mathrm{FeSi}_{2}$ signal decreases.

\section{Conclusions}

Short-period Fe/Si multilayers deposited onto (001)-oriented Si substrate by sputtering were characterized by AFM, Raman scattering, and Kerr rotation. An experimental evidence of a presence of an amorphous $\mathrm{FeSi}_{2}$ layer inside the spacers, which should contain nominally only amorphous $\mathrm{Si}$ was demonstrated. The scenario of the evolution of the structure, morphology, and of the magnetic properties of $\mathrm{Fe} / \mathrm{Si}$ multilayers with an increasing thickness of Fe layers was proposed. This scenario is able to explain satisfactorily all experimental observations and is in agreement with known properties of single, thin Fe layers.

\section{Acknowledgments}

This work was supported in part by the Center of Excellence for Photon, Electron, and Ion Advanced Methods for Natural Sciences (CEPHEUS) within the European Commission Contract G1MA-CT-2002-04017, by the Center of Excellence for Magnetic and Molecular Materials for Future Electronics within the European Commission Contract G5MA-CT-2002-04049, by ACI NR0095 NANODYNE research project from the French Ministry of National Education, and by the research grant No. PBZ/KBN/044/P03/2001 from the State Committee for Scientific Research (Poland).

\section{References}

[1] T. Luciński, P. Wandziuk, F. Stobiecki, B. Andrzejewski, M. Kopcewicz, A. Hütten, G. Reiss, W. Szuszkiewicz, J. Magn. Magn. Mater. 282, 248 (2004).

[2] W. Szuszkiewicz, K. Fronc, M. Baran, R. Szymczak, F. Ott, B. Hennion, E. Dynowska, W. Paszkowicz, J.B. Pelka, R. Zuberek, M. Jouanne, J.F. Morhange, J. Supercond. 16, 205 (2003).

[3] M. Walterfang, W. Keune, E. Schuster, A.T. Zayak, P. Entel, W. Sturhahn, T.S. Toellner, E.E. Alp, P.T. Jochym, K. Parliński, Phys. Rev. B 71, 035309 (2005).

[4] G. Garreau, S. Hajjar, J.L. Bubendorff, C. Pirri, D. Berling, A. Mehdaoui, R. Stephan, P. Wetzel, S. Zabrocki, G. Gewinner, S. Boukari, E. Beaurepaire, Phys. Rev. B 71, 094430 (2005). 